\title{
Total fluid intake of children and adolescents: cross-sectional surveys in 13 countries worldwide
}

\author{
Iris Iglesia $^{1}$ - Isabelle Guelinckx ${ }^{2}$ Pilar M. De Miguel-Etayo ${ }^{1}$ • \\ Esther M. González-Gil ${ }^{1} \cdot$ Jordi Salas-Salvadó $^{3,4} \cdot$ Stavros A. Kavouras $^{5}$. \\ Joan Gandy ${ }^{6,7} \cdot$ Homero Martínez ${ }^{8,9}$ • Saptawati Bardosono ${ }^{10}$ - Morteza Abdollahi ${ }^{11}$. \\ Esmat Nasseri $^{11} \cdot$ Agnieszka Jarosz $^{12} \cdot$ Guansheng Ma $^{13,14} \cdot$ Esteban Carmuega $^{15}$. \\ Isabelle Thiébaut $^{16,17} \cdot$ Luis A. Moreno ${ }^{1}$
}

Received: 2 March 2015 / Accepted: 30 May 2015 / Published online: 18 June 2015

(C) The Author(s) 2015. This article is published with open access at Springerlink.com

\begin{abstract}
Purpose To describe total fluid intake (TFI) according to socio-demographic characteristics in children and adolescents worldwide.

Methods Data of 3611 children (4-9 years) and 8109 adolescents (10-18 years) were retrieved from 13 crosssectional surveys (47\% males). In three countries, school classes were randomly recruited with stratified cluster sampling design. In the other countries, participants were randomly recruited based on a quota method. TFI (drinking
\end{abstract}

This article is part of a supplement supported by Danone Nutricia Research.

Electronic supplementary material The online version of this article (doi:10.1007/s00394-015-0946-6) contains supplementary material, which is available to authorized users.

Luis A. Moreno

lmoreno@unizar.es

1 GENUD (Growth, Exercise, NUtrition and Development) Research Group, Faculty of Health Sciences, Universidad de Zaragoza, Zaragoza, Spain

2 Hydration \& Health Department, Danone Research, Palaiseau, France

3 Human Nutrition Unit, Hospital Universitari de Sant Joan de Reus, Faculty of Medicine and Health Sciences, IISPV (Institut d'Investigació Sanitària Pere Virgili), Biochemistry Biotechnology Department, Universitat Rovira i Virgili, Reus, Spain

4 CIBERobn (Centro de Investigación Biomédica en Red Fisiopatología de la Obesidad y Nutrición), Institute of Health Carlos III, Madrid, Spain

5 Department of Health Human Performance and Recreation, University of Arkansas, Fayetteville, AR, USA

6 British Dietetic Association, Birmingham, UK water and beverages of all kinds) was obtained with a fluidspecific record over 7 consecutive days. Adequacy was assessed by comparing TFI to $80 \%$ of adequate intake (AI) for total water intake set by European Food Safety Authority. Data on height, weight and socio-economic level were collected in most countries.

Results The mean (SD) TFI ranged from [1.32 (0.68)] to [1.35 (0.71)] L/day. Non-adherence to AIs for fluids ranged from $10 \%$ (Uruguay) to $>90 \%$ (Belgium). Females were more likely to meet the AIs for fluids than males (4-9 years: $28 \%$, OR $0.72, p=0.002 ; 10-18$ years: $20 \%$, OR $0.80, p=0.001$ ), while adolescents were less likely to meet the AI than children (OR 1.645, $p<0.001$ in males and OR 1.625, $p<0.001$ in females).

Conclusions A high proportion of children and adolescents are at risk of an inadequate fluid intake. This risk is

7 School of Life and Medical Services, University of Hertfordshire, Hatfield, UK

8 RAND Corporation, Santa Monica, CA, USA

9 Hospital Infantil de Mexico Federico Gomez, Mexico City, Mexico

10 Department of Nutrition, Faculty of Medicine, Universitas Indonesia, Jakarta, Indonesia

11 Department of Nutrition Research, National Nutrition and Food Technology Research Institute, Faculty of Nutrition Sciences and Food Technology, Shahid Beheshti University of Medical Sciences, Tehran, Iran

12 National Food and Nutrition Institute, Warsaw, Poland

13 National Institute for Nutrition and Food Safety, Chinese Center for Disease Control and Prevention, Beijing, China

14 Department of Nutrition and Food Hygiene, School of Public Health, Peking University, Beijing, China 
especially high in males and adolescents when compared with females or children categories. This highlights water intake among young populations as an issue of global concern.

Keywords Fluid intake - Children · Adolescents . Worldwide $\cdot$ Dietary assessment $\cdot$ Hydration

\section{Introduction}

Water is essential for life to the extent that hydration is a major key to survival [24]. This is especially true for infants and adolescents who have relatively high requirements for water to maintain an adequate body composition [28]. This high requirement can partially be explained because children have proportionally higher body water content than adults [5]. Besides the higher body water content, body surface area to body mass ratio is higher in children when compared with adults. This difference levels out by adolescence, when children have almost reached their adult size [27]. At this time, gender differences start to appear: females store more adipose tissue than males and therefore water percentages are lower than in males [1].

Dehydration (body water deficit) is a physiologic state that can have profound implications for human health [10]. Under conditions of severe dehydration, a decreased sympathetic nervous activity, impaired thermoregulation and impaired cognitive and physical performance can be observed [29]. In children, even mild dehydration can affect cognitive school performance [16, 17].

International organizations have set dietary reference intakes (DRIs) for total water in young populations, using different methodologies. For instance, in children between the ages of 4 and 13, the European Food Safety Authority (EFSA) based its DRIs primarily upon energy intake, while for adolescents aged 14 years through to adulthood, intake reference values are based on population median water consumption and desirable urine osmolality [1]. The Institute of Medicine (IOM) DRIs for total water intake in the USA and Canada are the median intakes observed in the National Health and Nutrition Examination Survey III (NHANES), for children aged 1-18 years as well as for adults [2]. Due to such differences in methods for the derivation of reference values, health authorities stress the

15 Centro de Estudios Sobre Nutrición Infantil, Buenos Aires, Argentina

16 Research Centre of Epidemiology, Biostatistics and Clinical Research, School of Public Health, Université Libre de Bruxelles, Brussels, Belgium

17 Club Européen des Diététiciens de l'Enfance, Brussels, Belgium necessity to establish the water intake recommendations based on water balance [1].

Despite the availability of intake reference values specific for children and adolescents, little is known about the adherence to these recommendations in these age groups. The limited data available suggest that children and adolescents do not drink enough and do not meet the daily recommended fluid intake. Total fluid intake (TFI) is set as the sum of liquids provided by all types of fluids or beverages, and it is assumed to be around $80 \%$ of all the water intakes (20\% from foods) [1]. A German longitudinal study conducted in children and adolescents reported a mean total water intake of 1642 and $1457 \mathrm{ml}$ in 9-13-year-old males and females, respectively [4]. This is approximately $450 \mathrm{ml}$ lower than the corresponding EFSA reference values of 2100 (males) and $1900 \mathrm{ml}$ (females) [26]. A similar situation was observed in European adolescents, as their mean total water intake was 1611 and $1316 \mathrm{ml}$ in 12.5-17.5-year-old males and females, respectively [15]. In US children and adolescents, 4-19 years in the NHANES survey 2005-2006, mean daily total water intake was lower than the IOM adequate intake (AI) (only 15-35\% met the recommendations according to sex and age groups) [21]. In a Brazilian study [18], in which no statistically significant differences were observed between males and females, TFI was higher than that observed in the previously cited studies, being approximately $1750 \mathrm{ml}$ for children and $2050 \mathrm{ml}$ for adolescents.

The aim of the present study is to describe fluid intake and its variation by age (children $4-9$ years vs. adolescents 10-18 years) and/or sex in 13 countries worldwide controlling for BMI and socio-economic status.

\section{Methods}

\section{Design and study population}

Cross-sectional surveys were identified from 13 different countries to summarize country-specific TFI of participants aged 4-18 years. The surveys (whose aim was to systematically obtain a complete set of data from fluid intake) were conducted during one period of the year in Latin America (Mexico, Brazil, Argentina, Uruguay), Europe (Spain, France, Belgium, UK, Poland, Turkey) and Asia (Iran, China, Indonesia) by private research organizations, the Université libre de Bruxelles/the Club Européen des Diététiciens de l'Enfance (CEDE), the Iranian National Nutrition \& Food Technology Research Institute (NNFTRI) and the Chinese Center for Disease Control (CDC). The individual surveys took place between 2008 and 2014 (Online Resource 1).

The protocol of the published surveys was described in detail elsewhere [13, 14, 25], but will again briefly be described. The surveys performed in Belgium, Iran and 
China had a comparable method of recruitment: they recruited entire school classes via a random, stratified cluster sampling. In Belgium, 13 schools had accepted to participate, and in each school, the classes of the third- up to the sixth-grade participated [25]. In Iran, the recruitment was performed in Tehran. To cover all SES groups, Tehran was divided into three major areas: north, middle and south, representing high, middle and low SES, respectively. Eighteen schools were randomly selected to cover three school levels and two genders in every area. In selected schools, one class was randomly selected from each grade (except for the first and 12th grades). All of the students of a class were recruited. In China, a multi-stage random sampling method is adopted throughout the survey [13, 14]. The parents of the recruited school children were informed via meetings, written information sheets or phone calls. The surveys performed in the 10 other countries randomly recruited participants using a quota-based method with quotas set for age, gender, region of the country, habitat and/or socio-economic characteristics. The parents of the children were contacted via a database of individuals volunteering for population surveys or via a systematic doorto-door approach, with an invitation for their child to participate. Having a parent working in advertising, marketing, market research, media or manufacture, distribution and sale of beverages (in order to have participants that were not specifically aware of their fluid intake) and being incapable of completing the questionnaire in the language presented were exclusion criteria. Having a specific diagnosed disease and/or following a medically prescribed diet were additional exclusion criteria (to avoid individuals that might have modified their usual fluid intake) in the surveys of UK and China. The surveys in Argentina, Poland and Japan also excluded participants who took part in a survey about non-alcoholic drinks in the last 6 months.

All parents and children willing to participate (those who did not were not tracked) in the survey received detailed information about the survey objectives, what was expected from them, as well as a disclosure of the survey's provisions to preserve confidentiality, risks and benefits, and a clear explanation about their option to voluntarily participate or not in the survey. After offering a fullinformed description of the survey, parents were asked for their oral approval to let their child participate. No monetary incentive was offered for taking part in the survey. All data were recorded in an anonymous way. Therefore, participants cannot be identified, directly or through identifiers linked to the participants. The protocol of the unpublished surveys was reviewed and approved by the Institutional Review Board, Office of Research Compliance of the University of Arkansas (IRB Protocol \# 14-12-376).

\section{Assessment of fluid intake}

A fluid-specific record over 7 consecutive days was completed by the participants. These 7-day fluid-specific records and the associated written information were presented to the participants in the official language of the country and were in a paper format, except in France where the fluid records were completed online. This online fluidspecific record, which had the same structure as the paper version, was supported with paper memory cards to make notes throughout the day. An investigator delivered and explained the fluid record to the participants during a faceto-face interview at home. For children below 12 years, the primary caregiver of the child was asked to complete the fluid records. The researcher visited the home again after 7 days to collect the fluid-specific records and to ensure their completion. The surveys performed in Belgium, Iran and China deviated from this protocol as they recruited children in school classes $[3,5]$. In these cases, both parents and teachers were involved in the completion of the fluid records.

The 7-day fluid-specific records in all surveys were structured in the same way in order to capture the type of fluid consumed, the volume of intake, the reasons, the moment of the day and the place of all fluid intakes. To assist the participants in estimating the consumed volumes, the records were supported by a photographic booklet of standard containers of fluids and in China by an additional scaled water container. The sum of all fluid types recorded was defined as TFI. To evaluate the adequacy of fluid intake, the EFSA age- and gender-specific reference values for total water intake were used in order not to overestimate the size of the problem of inadequate fluid intake (in comparison with other reference values), after extracting $20 \%$ supposed to correspond to water intake from foods [1]. Consequently the reference values of 1.3, 1.7 and 2 L/day were used for males aged 4-8, 9-13 and 14-18 years, respectively. The reference values of $1.3,1.5$ and $1.6 \mathrm{~L} /$ day were used for females aged 4-8, 9-13 and 14-18 years respectively. These values will be referred to as EFSA AIs for fluids. However, the presentation of the results throughout the paper will be done only by children and adolescents, but fitting them in their corresponding reference value for water intake recommendations.

\section{Assessment of body composition and socio-economical level}

Body mass index (BMI) serves as a proxy for both low physical activity and poor health status, two key determinants of water requirements. Height in metres (m) and weight in kilograms $(\mathrm{kg})$ were self-reported in the surveys in Spain, France, UK and Turkey and measured 
by the investigator in the surveys in Belgium, Poland, Iran and China. No anthropometric data were collected in Mexico, Brazil, Uruguay, Argentina and Indonesia. Body mass index (BMI) was calculated $\left(\mathrm{kg} / \mathrm{m}^{2}\right)$ and categorized using the International Obesity Task Force cutoff points $[8,9]$. These cut-off points were established according to thinness, sex and age (i.e. male, 8 years, BMI $14.14 \mathrm{~kg} / \mathrm{m}^{2}$ or lower $=$ underweight; male, 8 years, BMI $=14.15-18.43 \mathrm{~kg} / \mathrm{m}^{2}=$ normal weight; male, 8 years, BMI $=18.44-21.59 \mathrm{~kg} / \mathrm{m}^{2}=$ overweight; male, 8 years, BMI $=21.60 \mathrm{~kg} / \mathrm{m}^{2}$ or higher $=$ obesity). Socioeconomic level (SEL) was assessed using a self-administered questionnaire in most of the countries and was categorized using the Market Research Society classification. This classification was based on the occupation of the chief income earner in the household $[3,12]$.

\section{Statistical analysis}

Participants who did not complete the full 7-day fluid intake records or participants reporting a mean total daily fluid intake below $0.4 \mathrm{~L} /$ day or higher than $4 \mathrm{~L}$ /day were excluded from the analysis. The final sample size for this analysis was 11720 participants. Continuous and categorical data were, respectively, presented as mean (SD) and/or median (25th-75th percentile) and percentage $(n)$. Standard error of the mean (SEM) and additional percentiles (5th, 10th, 90th, 95th) are reported in Online Resource 2. The effect of gender on the non-adherence to AIs for total water intake was tested with Chi-square test stratified by country.

Associations between compliance with AIs for fluids by gender and age group were assessed by logistic regression analysis (Fig. 2) stratifying the results by country. To estimate the strength of the association, odds ratios and $95 \%$ confidence intervals (CI) were assessed using age- or gender-stratified forest plots. The models were adjusted for gender, BMI and SEL. Males were considered as reference in the first model (Fig. 2). Indonesia, Mexico, Argentina, Brazil and Uruguay were excluded from the logistic regression analysis due to the lack of data for some covariates. Analyses were performed using SPSS software version 20.0 (SPSS Inc, Chicago, IL). All statistical tests were twotailed, and the significance level was set at $p<0.05$.

\section{Results}

\section{Sample description}

The general characteristics of both male and female subjects are presented by country in Tables 1 and 2. A total of 5766 males $(11.2 \pm 3.3$ years $)$ and 6333 females $(11.4 \pm 3.3$ years $)$ were included in this study. In only 8 out of 13 countries, anthropometric data were obtained, and consequently the BMI of only 3934 males and 4498 females was calculated, and $64.5 \%$ males and $67.8 \%$ females of these were classified as normal weight. Medium socio-economic class was the most common SEL (44.0\% males and $47.0 \%$ females).

China contributed the highest number of males to the total male study sample (Table 1$)(n=2705,47 \%$ of total male study sample) and the lowest Turkey ( $n=67$, $1 \%$ of total male sample). The youngest females were from France $(8.9 \pm 3.4$ years $)$ and the oldest were from Iran (13.0 \pm 4.1 years). China provided the highest number of females (Table 2) $(n=2922)$ and Uruguay the lowest $(n=71)$. The youngest females were from Mexico (9.4 \pm 3.7 years) and the oldest were from Iran $(13.0 \pm 3.0$ years).

\section{Total fluid intake}

Daily TFI was obtained in 3611 children (4-9 years, $51.75 \%$ females) and 8109 adolescents (10-17 years, $53.11 \%$ females) (Table 3). The highest fluid intake was observed in Uruguayan males $(2.13 \pm 0.80$ and $2.46 \pm 1.04 \mathrm{~L} /$ day, for $4-9$ years and 10-18 years, respectively) and females $(2.47 \pm 0.92$ and $2.61 \pm 1.16 \mathrm{~L} /$ day, for 4-9 years and 10-18 years, respectively). In contrast, the lowest fluid intake in children and adolescents was observed in Belgian males $(0.90 \pm 0.43$ and $0.99 \pm 0.44 \mathrm{~L} /$ day, for 4-9 and 10-18 years, respectively) and females $(0.73 \pm 0.39$ and $0.91 \pm 0.41 \mathrm{~L} /$ day, respectively $)$.

\section{Adherence to EFSA reference values}

The non-adherence to EFSA AIs for fluids in children and adolescents is shown in Fig. 1. A high proportion of the participants do not meet the EFSA AIs for fluids. In children, the lowest non-adherence was observed in Uruguay, males $(10 \%)$ and females $(<20 \%)$, matching with the country which provided fewer participants, and the highest non-adherence was observed in Belgium, males and females $(>90 \%)$. No significant differences for adherence were observed between males and females in all countries.

In adolescents, the lowest non-adherence to EFSA AIs for fluids was observed in Uruguay (males and females $<25$ and $15 \%$, respectively) and the highest non-adherence was observed in Belgium (males and females $>90 \%$ ). Nonadherence to EFSA AIs for fluids was significantly higher in males than in females in Indonesia, Brazil, Argentina, Mexico and Poland.

In Figs. 2 and 3, compliance with EFSA AIs for fluids (yes/no) is considered as the dependent variable and gender as the independent variable. The odds ratios (OR) represented the likelihood of not reaching the EFSA AIs for 


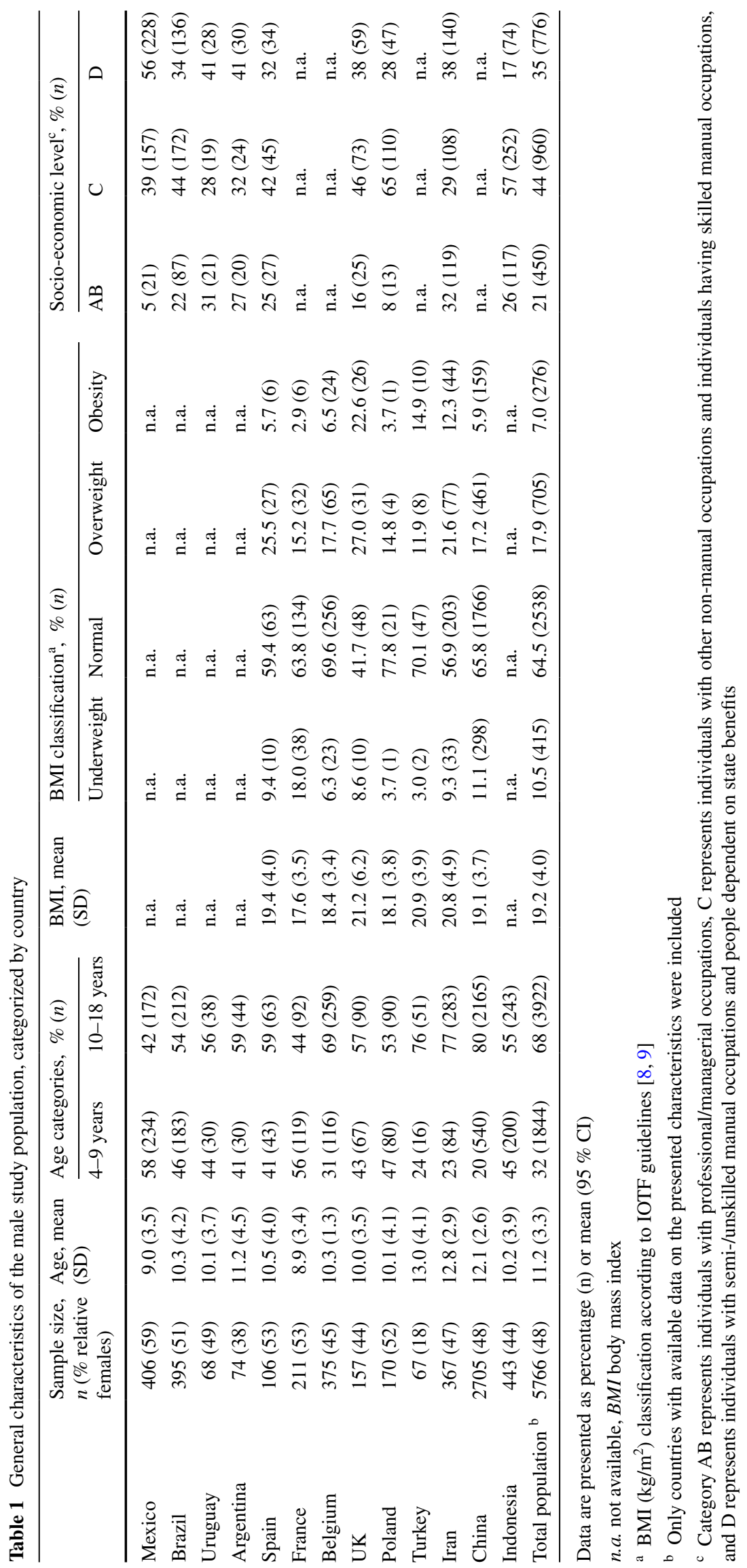







Table 3 Daily total fluid intake (the sum of liquids provided by all types of fluids or beverages) (L/day) per age group, sex and country

\begin{tabular}{|c|c|c|c|c|c|c|c|c|c|c|c|c|c|c|c|c|c|c|}
\hline & \multicolumn{6}{|l|}{ Total } & \multicolumn{6}{|c|}{ Males } & \multicolumn{6}{|c|}{ Females } \\
\hline & $n$ & Mean & SD & Median & 25 th & 75th & $n$ & Mean & SD & Median & 25th & 75th & $n$ & Mean & SD & Median & 25th & 75th \\
\hline \multicolumn{19}{|c|}{ Children (4-9 years) } \\
\hline Mexico & 387 & 1.35 & 0.65 & 1.23 & 0.88 & 1.64 & 234 & 1.39 & 0.65 & 1.27 & 0.89 & 1.71 & 153 & 1.28 & 0.64 & 1.16 & 0.84 & 1.51 \\
\hline Brazil & 349 & 1.67 & 0.63 & 1.55 & 1.20 & 2.03 & 183 & 1.68 & 0.61 & 1.55 & 1.23 & 2.10 & 166 & 1.66 & 0.66 & 1.55 & 1.20 & 1.94 \\
\hline Uruguay & 60 & 2.24 & 0.86 & 2.05 & 1.70 & 2.72 & 30 & 2.13 & 0.80 & 2.00 & 1.53 & 2.72 & 30 & 2.35 & 0.92 & 2.16 & 1.86 & 2.78 \\
\hline Argentina & 89 & 1.78 & 0.92 & 1.54 & 1.17 & 2.03 & 30 & 1.64 & 0.63 & 1.58 & 1.23 & 1.99 & 59 & 1.86 & 1.03 & 1.50 & 1.14 & 2.20 \\
\hline Spain & 85 & 1.64 & 0.67 & 1.50 & 1.13 & 1.97 & 43 & 1.78 & 0.77 & 1.68 & 1.18 & 2.08 & 42 & 1.50 & 0.53 & 1.43 & 1.09 & 1.73 \\
\hline France & 206 & 1.02 & 0.35 & 0.92 & 0.77 & 1.22 & 119 & 1.02 & 0.35 & 0.95 & 0.77 & 1.23 & 87 & 1.01 & 0.36 & 0.88 & 0.77 & 1.21 \\
\hline Belgium & 266 & 0.84 & 0.41 & 0.81 & 0.54 & 1.08 & 116 & 0.90 & 0.43 & 0.90 & 0.60 & 1.16 & 150 & 0.79 & 0.39 & 0.75 & 0.49 & 1.03 \\
\hline UK & 148 & 1.56 & 0.57 & 1.51 & 1.18 & 1.87 & 67 & 1.69 & 0.64 & 1.65 & 1.24 & 2.08 & 81 & 1.45 & 0.49 & 1.46 & 1.13 & 1.71 \\
\hline Poland & 154 & 1.39 & 0.44 & 1.29 & 1.07 & 1.64 & 80 & 1.42 & 0.50 & 1.28 & 1.06 & 1.69 & 74 & 1.37 & 0.38 & 1.29 & 1.09 & 1.64 \\
\hline Turkey & 164 & 1.74 & 0.72 & 1.67 & 1.23 & 2.12 & 16 & 1.81 & 0.75 & 1.84 & 1.06 & 2.31 & 148 & 1.73 & 0.71 & 1.60 & 1.23 & 2.04 \\
\hline Iran & 177 & 1.24 & 0.42 & 1.19 & 0.95 & 1.46 & 84 & 1.32 & 0.44 & 1.25 & 1.02 & 1.47 & 93 & 1.16 & 0.39 & 1.13 & 0.87 & 1.41 \\
\hline China & 1120 & 0.97 & 0.43 & 0.89 & 0.64 & 1.19 & 540 & 0.98 & 0.42 & 0.90 & 0.65 & 1.20 & 580 & 0.95 & 0.43 & 0.86 & 0.63 & 1.17 \\
\hline Indonesia & 406 & 1.88 & 0.77 & 1.73 & 1.26 & 2.44 & 200 & 1.94 & 0.78 & 1.82 & 1.36 & 2.55 & 206 & 1.83 & 0.77 & 1.68 & 1.19 & 2.29 \\
\hline TOTAL & 3611 & 1.32 & 0.68 & 1.18 & 0.84 & 1.64 & 1742 & 1.34 & 0.67 & 1.20 & 0.86 & 1.67 & 1869 & 1.30 & 0.69 & 1.16 & 0.81 & 1.62 \\
\hline \multicolumn{19}{|c|}{ Adolescents (10-18 years) } \\
\hline Mexico & 306 & 1.51 & 0.85 & 1.31 & 0.91 & 1.91 & 172 & 1.46 & 0.74 & 1.33 & 0.96 & 1.81 & 134 & 1.57 & 0.97 & 1.29 & 0.84 & 2.05 \\
\hline Brazil & 430 & 2.01 & 0.90 & 1.80 & 1.40 & 2.54 & 212 & 2.03 & 0.92 & 1.89 & 1.35 & 2.55 & 218 & 1.99 & 0.88 & 1.75 & 1.40 & 2.48 \\
\hline Uruguay & 79 & 2.54 & 1.04 & 2.23 & 1.75 & 3.16 & 38 & 2.46 & 0.92 & 2.21 & 1.77 & 3.04 & 41 & 2.61 & 1.16 & 2.25 & 1.75 & 3.42 \\
\hline Argentina & 104 & 1.77 & 0.81 & 1.59 & 1.21 & 2.11 & 44 & 1.83 & 0.97 & 1.57 & 1.17 & 2.24 & 60 & 1.72 & 0.68 & 1.63 & 1.26 & 2.07 \\
\hline Spain & 116 & 1.78 & 0.70 & 1.62 & 1.33 & 2.14 & 63 & 1.80 & 0.64 & 1.63 & 1.36 & 2.15 & 53 & 1.75 & 0.76 & 1.57 & 1.27 & 2.15 \\
\hline France & 193 & 1.25 & 0.45 & 1.16 & 0.93 & 1.47 & 92 & 1.35 & 0.46 & 1.28 & 1.04 & 1.57 & 101 & 1.17 & 0.43 & 1.07 & 0.91 & 1.32 \\
\hline Belgium & 574 & 0.95 & 0.43 & 0.91 & 0.69 & 1.14 & 259 & 0.99 & 0.44 & 0.95 & 0.71 & 1.18 & 315 & 0.91 & 0.41 & 0.86 & 0.65 & 1.09 \\
\hline UK & 210 & 1.67 & 0.73 & 1.55 & 1.15 & 2.09 & 90 & 1.77 & 0.75 & 1.61 & 1.23 & 2.16 & 120 & 1.60 & 0.72 & 1.45 & 1.10 & 1.96 \\
\hline Poland & 176 & 1.48 & 0.48 & 1.41 & 1.12 & 1.73 & 90 & 1.44 & 0.46 & 1.42 & 1.10 & 1.69 & 86 & 1.51 & 0.51 & 1.39 & 1.14 & 1.93 \\
\hline Turkey & 212 & 1.91 & 0.80 & 1.77 & 1.26 & 2.46 & 51 & 1.98 & 0.86 & 1.91 & 1.16 & 2.77 & 161 & 1.88 & 0.78 & 1.72 & 1.31 & 2.34 \\
\hline Iran & 607 & 1.32 & 0.51 & 1.26 & 0.94 & 1.58 & 283 & 1.42 & 0.53 & 1.36 & 1.00 & 1.70 & 324 & 1.23 & 0.47 & 1.16 & 0.86 & 1.52 \\
\hline China & 4507 & 1.15 & 0.54 & 1.05 & 0.76 & 1.43 & 2165 & 1.24 & 0.57 & 1.13 & 0.81 & 1.53 & 2342 & 1.08 & 0.49 & 0.99 & 0.72 & 1.33 \\
\hline Indonesia & 595 & 2.03 & 0.83 & 1.91 & 1.37 & 2.66 & 243 & 2.05 & 0.86 & 1.97 & 1.34 & 2.75 & 352 & 2.01 & 0.81 & 1.90 & 1.38 & 2.62 \\
\hline Total & 8109 & 1.35 & 0.71 & 1.19 & 0.84 & 1.65 & 3802 & 1.40 & 0.71 & 1.25 & 0.90 & 1.73 & 4307 & 1.30 & 0.70 & 1.13 & 0.80 & 1.59 \\
\hline
\end{tabular}

fluids when being female as compared to being male. In the total sample, females were more likely to meet the EFSA AIs for fluids than the males (4-9 years: $28 \%$, OR 0.72 , $p=0.002$; $10-18$ years: $20 \%$, OR $0.80, p=0.001)$. When analysing the samples of the countries individually, the probability of not meeting the EFSA AIs for fluids reached significance only in Spain and Poland, and moreover only in the age group 10-18 years: females were more likely to meet the EFSA AIs for fluids than males (Spain: $57 \%$, OR 0.43, $p=0.041$; and Poland: $62 \%$, OR 0.38, $p=0.010$ ).

In Figs. 4 and 5, compliance with EFSA recommendation for TFI (yes/no) is considered as dependent variable and age 4-9 as independent variable. The odds ratio (OR) represents the percentage not reaching the EFSA recommendation for TFI when being older than 9 years.
Differences were found between country and gender (males Fig. 4; females Fig. 5). The probability of noncompliance with EFSA AI for fluids increased significantly when being older than 9 years in Spain (OR 3.345, $p=0.021$ in males), UK (OR 2.689, $p=0.019$, in males), Poland (OR 4.871, $p<0.001$ in males), Iran (OR 3.747, $p<0.001$ in males and OR 2.702, $p=0.003$ in females) and the total sample (OR 1.645, $p<0.001$ in males and OR $1.625, p<0.001$ in females). In the rest of the countries except for China, children older than 9 years were more likely to comply with the EFSA AIs; however, this was not significant. In China, the probability of not compliance with EFSA recommendation was lower when being older than 9 years in both genders, although this did not reach significance. 

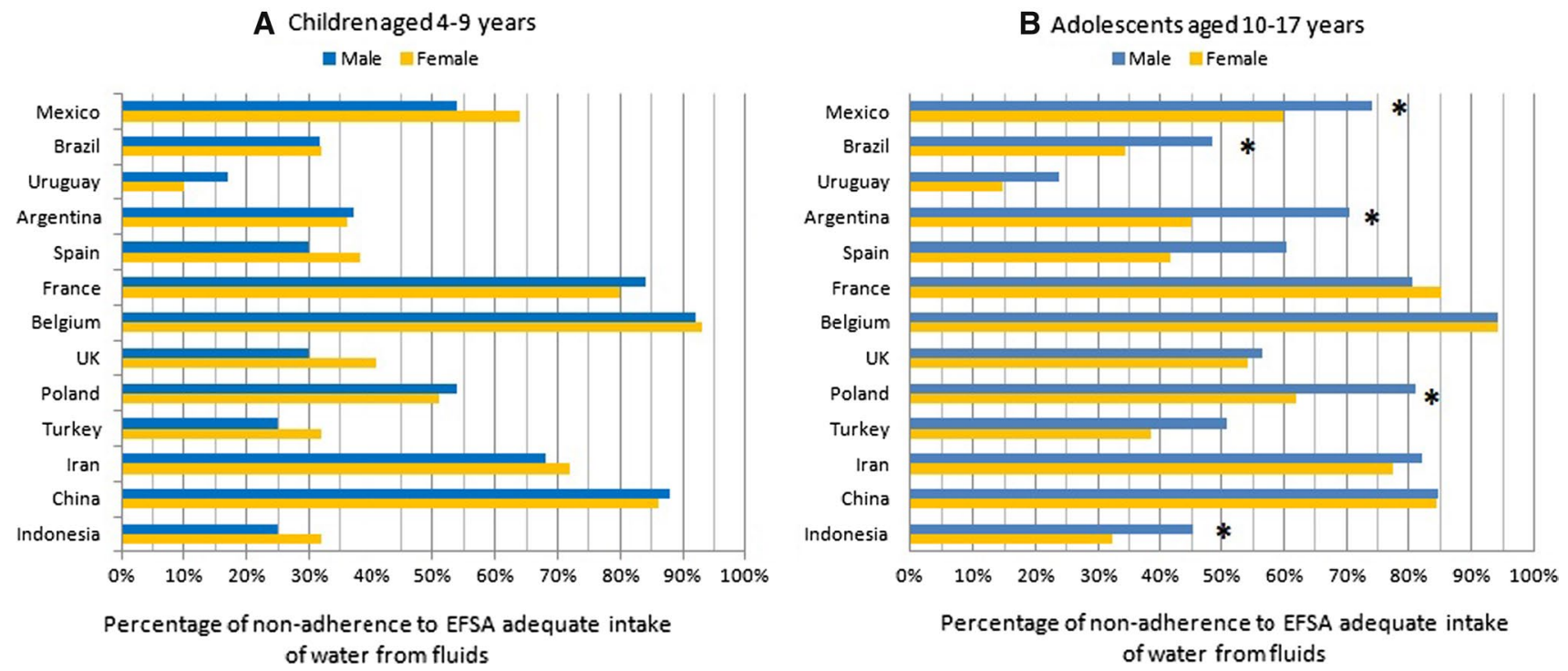

Fig. 1 Non-adherence to EFSA adequate intake for fluids (equals $80 \%$ of EFSA adequate intake for total water intake) by age group. $* p$ value of the Pearson Chi-square test $<0.05$ (differences between sexes within countries)

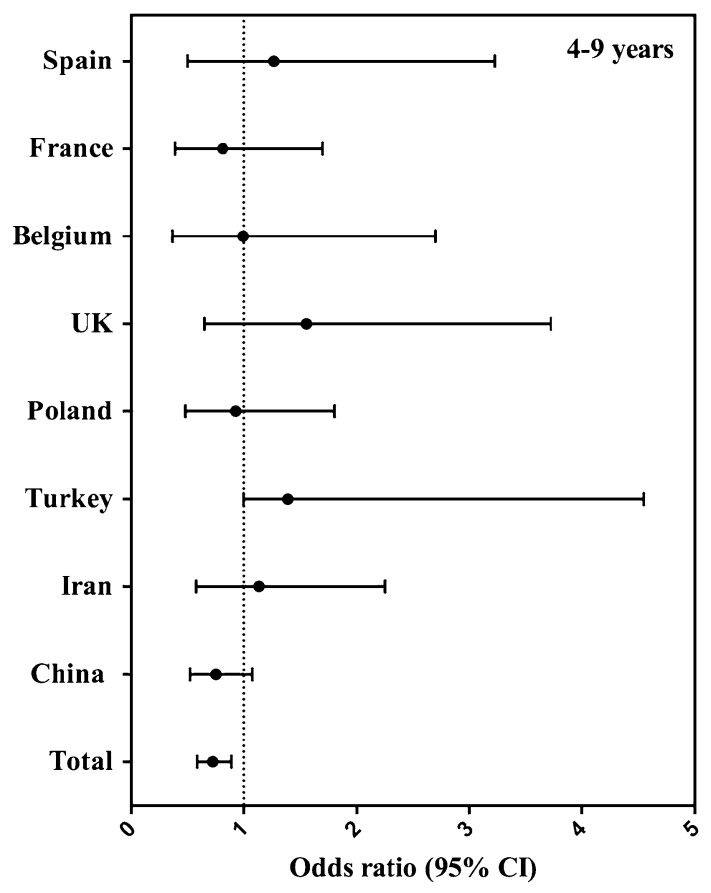

Fig. 2 Association (odds ratio, $95 \% \mathrm{CI}$ ) between compliance with EFSA adequate intake for fluids (equals $80 \%$ of EFSA adequate intake for total water intake) and gender, per country in children aged 4-9 years. The logistic regression model was adjusted for age, BMI and SEL, and males were considered as reference. $* p$ value $<0.05$

\section{Discussion}

The results of this compilation of cross-sectional studies provide important information about the fluid intake of

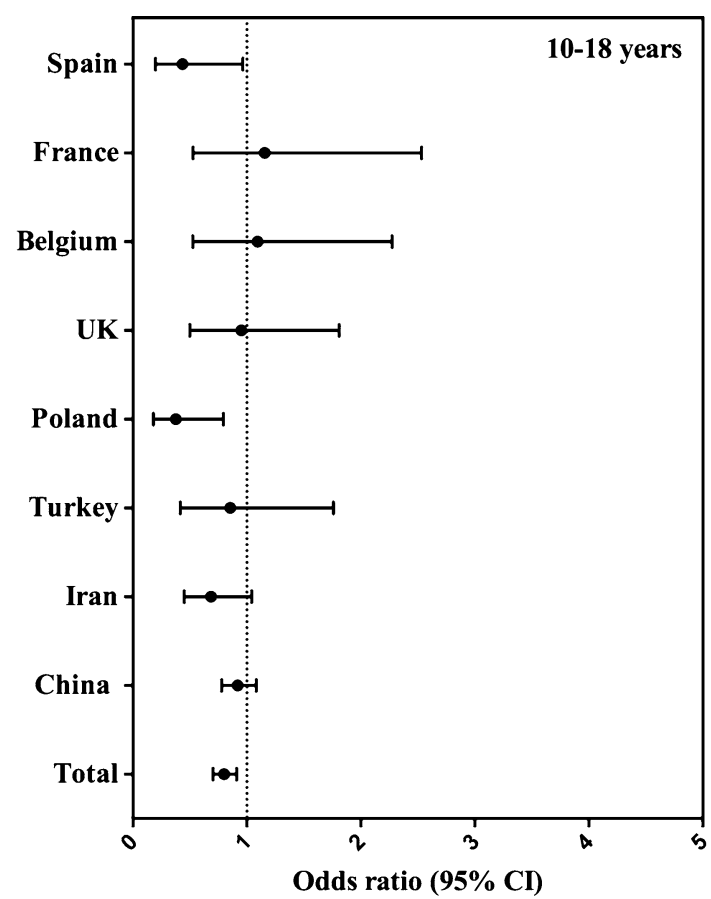

Fig. 3 Association (odds ratio, $95 \% \mathrm{CI}$ ) between compliance with EFSA adequate intake for fluids (equals $80 \%$ of EFSA adequate intake for total water intake) and gender, per country in adolescents aged 10-17 years. The logistic regression model was adjusted for age, $\mathrm{BMI}$ and SEL, and males were considered as reference

children in 13 countries, and such a global perspective has not previously been reported. According to the EFSA reference values for total water intake for these age categories, 


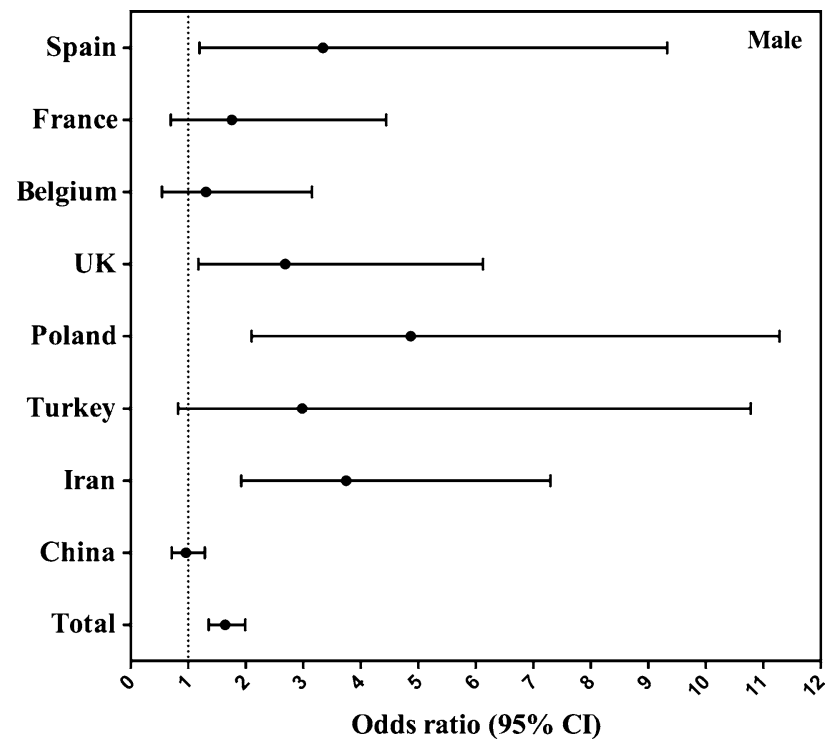

Fig. 4 Association (odds ratio, $95 \% \mathrm{CI}$ ) between compliance with EFSA adequate intake for fluids (equals $80 \%$ of EFSA adequate intake for total water intake) and age group per country in males. The logistic regression model was adjusted for BMI and SEL, and children aged 4-9 were considered as reference

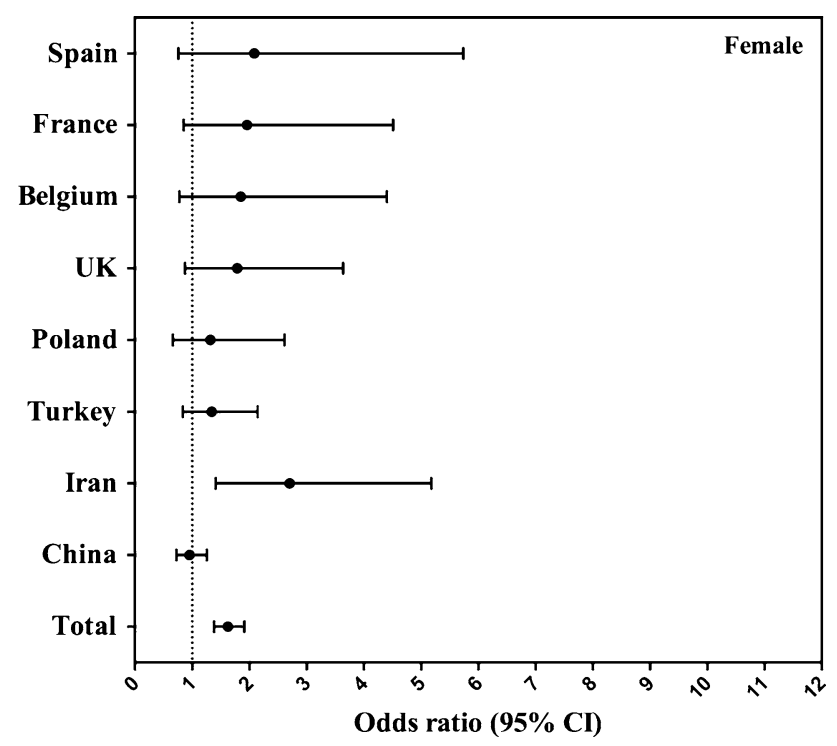

Fig. 5 Association (odds ratio, $95 \% \mathrm{CI}$ ) between compliance with EFSA adequate intake for fluids (equals $80 \%$ of EFSA adequate intake for total water intake) and age group per country in females. The logistic regression model was adjusted for BMI and SEL, and children aged 4-9 were considered as reference

more than fifty per cent of the whole study population are at risk of an inadequate intake.

Large differences between countries and age groups were found. Belgium had the highest percentage (>90\%) of non-adherence to the EFSA AIs for fluids in both children and adolescents, followed by China, France (both $>80 \%$ ) and Iran (70 \%). In Belgium, China and Iran, the completion of the fluid diary was done by the teachers in the school and by the parents at home, whereas in the rest of the countries, the diary was filled by main caretaker (mums). This fact may explain the lower intakes recorded in the firsts countries. Maybe the intake in these other countries was overestimated because of social desirability and is the intake of Belgium actually more accurately reflecting the true intake. In France, a study [6] with a representative sample of children and adolescents suggested that fluid intake was low (1-1.1 L/day), very similar to the values obtained in the present study. Moreover, another study demonstrated that based on osmolality urine levels, two-thirds of French children had a hydration deficit in the morning when they went to school, despite having breakfast [7]. Both these studies support our findings of an inadequate fluid intake in French children. In Europe, data from the HELENA study [15] performed in adolescents, and the German DONALD study [26] performed in both children and adolescents, suggested higher values of fluid intakes than our study did. However, the tool used in the HELENA study was not specifically developed to measure fluid intake [15]. In addition, the software used in the HELENA study to collect the 24-h recalls (HELENA-DIAT [11]) was designed to remind the adolescents to fulfil the questionnaire to state the amount of water intake as is an issue susceptible to oversight such as other add-ons like butter, or salad dressing.

Uruguay $(<20 \%)$ and Brazil $(<40 \%)$ had the lowest percentages of non-adherents to EFSA AIs for fluids. Together with Argentina, Turkey and Indonesia, Uruguay had the highest mean fluid intakes, similar to the values observed in a previous study in Brazil [18]. One of the plausible explanations for this finding could be the high humidity and temperatures in these countries, which result in people drinking more [30]. This raises one of the limitations of present analysis of cross-sectional surveys. The fact that the surveys were performed in the same period of the year, and therefore the impact of the seasons on TFI was neglected. Nevertheless, the data collection was performed during the spring or autumn, periods expected to have a mild climate. The impact of climate on TFI should be investigated by recording the temperature and humidity in future surveys and considered in analyses.

One remarkable point throughout this analysis is that there were no large differences in fluid consumption between the genders. Generally males drank more fluids than females in all the countries included in this survey (except females from Uruguay and Argentina aged 4-10 years, and females from Mexico and Uruguay aged 10-18 years). However, females were more likely to comply with EFSA AIs for fluids. Gender differences were 
more noticeable in the adolescents throughout the countries and in the total sample, probably because at these ages, females become more health conscious and may drink more [23].

The results also indicated that compliance with EFSA AIs for fluids is greater among children 4-9 years old than among children 10-18 years old in almost all included countries. A plausible explanation could be that with transition to adolescence children gain more independence with respect to food/fluid consumption. While they still consume the majority of meals and snacks at home, but they also start taking decisions regarding food choices away from home [22]. With the transition to adolescence, a possible transition in the preference for certain beverage types might be anticipated. This topic was, however, outside the scope of the current paper, but will be addressed in a separate paper [20].

Several limitations should be considered when interpreting the data presented. Firstly, the lack of representativeness of the samples in terms of " $n$ ", age groups and gender within the countries suggests that these data should be interpreted cautiously: as country-, age- and gender-specific data points. Secondly, the lacks of data regarding BMI or SEL in some of the countries did not allow having a complete set up of the situation. Another limitation to consider is related to the method used for recording fluid intake: for children younger than 12 years, the primary caretaker was responsible to record the fluid intake of the child. It should be considered that a parent might find it difficult to accurately estimate fluid intake consumed at school. Therefore, fluid intake of the children/adolescent could be over- or underreported. However, fluid intake of all participants was recorded over a period of 7 days including the weekend during which the children are at home and the caregiver can observe and record the intake of their child more carefully. Future research should focus on demonstrating the health impact of a certain level of fluid intake, in order to progress towards a reference value based on scientific evidence and not only on observed intakes. Regardless of which cut-off was used to evaluate the wide of the problem (meet/not meet water intake recommendations), the observation from these cross-sectional surveys remains the same: that a high number of children and adolescents worldwide have a low TFI. For example, when using the dietary reference values set in China (which use those established by the Institute of Medicine-IOM-in USA [2]), $86 \%$ of both males and females were at risk of an inadequate water intake, which is a very similar percentage to the ones we have obtained based on the EFSA recommendations even when the values established by the IOM are higher than the ones from EFSA. This is because there are almost no subjects located between the EFSA reference values and IOM ones, regarding drinking fluids.
The strengths of all 13 cross-sectional surveys include the use of a standardized 7-day fluid-specific record, considered the reference method to assess fluid intake and the amount of water volumes also in young population groups [31, 32]. This fluid-specific record was also supported by visual aids, to facilitate the recording of consumed volumes. Moreover, the two methods used for the recruitment (the quota-based sample-the best method to evaluate intakes [19] —and stratified clusterization) used to approach the survey in 10 and in the other three countries, respectively, are recognized as valuable methods to provide enough sample by age of participants, regions of the country and different socio-economic groups for meaningful analysis.

\section{Conclusions}

The most important conclusions from this analysis are that a high proportion of children and adolescents are at risk of an inadequate fluid intake and that the probability for non-adherence to EFSA AIs for fluids is higher among males than among females, and among those aged from 4 to 9 than among those aged from 10 to 18 . Uruguay followed by Brazil and Belgium followed by China were those countries with the highest proportion of adherents and non-adherents, respectively, to EFSA AIs for fluids. As a number of studies have demonstrated that having a low fluid or water intake can compromise several body functions, this conclusion justifies encouraging these populations to increase their plain water intake. Future studies should focus on the observation of longitudinal changes to determine whether the maintained restrictive water intake can result in long-term health impacts from the early stages of life.

Acknowledgments Data collection was performed by the Center of Disease Control in China; the National Nutrition and Food Technology Research Institute in Iran; the School of Public Health, Université Libre de Bruxelles and the Club Européen des Diététiciens de l'Enfance in Belgium. We acknowledge Christine Jean, Market Research \& Consumer Intelligence Danone Waters and the entire MRCI Team to raise the methodology of fluid intake surveys to the current standard and to guide IPSOS in Mexico, UK and Turkey; TNS in Argentina, France, Germany, Poland and Spain; Nielsen in Indonesia; and GFK in Brazil and Uruguay, with the preparation of the survey.

Conflict of interest IG is an employee of Danone Research. JS-S, JS, LAM, SK, JG, HM are members of advisory board on fluid intake of Danone Research. AM and NE are employer at NNFTRI, an institute which has received a research grant from Damavand mineral water company (a brand of Danone Group in Iran).

Ethical Standard The protocol of the unpublished surveys was reviewed and approved by the Institutional Review Board, Office of 
Research Compliance of the University of Arkansas (IRB Protocol \# 14-12-376). The Belgian survey was approved by the ethical committee of the Queen Fabiola Children's University Hospital of Brussels approved (on 20 September 2011) (reference: CEH-N ${ }^{\circ} 58 / 11$ ). The Chinese surveys were approved by the Ethical Review Committee of the National Institute for Nutrition and Food Safety, Chinese Center for Disease Control and Prevention. Informed consent was obtained by all participating children or adolescents and their parents, as appropriate. All data were recorded anonymously.

Open Access This article is distributed under the terms of the Creative Commons Attribution 4.0 International License (http://creativecommons.org/licenses/by/4.0/), which permits unrestricted use, distribution, and reproduction in any medium, provided you give appropriate credit to the original author(s) and the source, provide a link to the Creative Commons license, and indicate if changes were made.

\section{References}

1. European Food Safety Authority (EFSA) (2010) Scientific opinion on dietary reference values for water EFSA panel on dietetic products, nutrition, and allergies (NDA). EFSA J 8:1459

2. Institute of Medicine. Food and Nutrition Board (2004) Dietary reference intakes for water, potassium, sodium, chloride, and sulfate. National Academies Press, Washington, DC

3. The Market Research Society (2010) Occupation groupings: a job dictionary, 7edn. Market Research Society, London

4. Alexy U, Cheng G, Libuda L, Hilbig A, Kersting M (2012) $24 \mathrm{~h}$-Sodium excretion and hydration status in children and adolescents-results of the DONALD Study. Clin Nutr 31:78-84

5. Altman PL (1961) Blood and other body fluids. In: Federation of American Societies for Experimental Biology (FASEB), Washington, DC

6. Bellisle F, Thornton SN, Hebel P, Denizeau M, Tahiri M (2010) A study of fluid intake from beverages in a sample of healthy French children, adolescents and adults. Eur J Clin Nutr 64:350-355

7. Bonnet F, Lepicard EM, Cathrin L, Letellier C, Constant F, Hawili N, Friedlander G (2012) French children start their school day with a hydration deficit. Ann Nutr Metab 60:257-263

8. Cole TJ, Bellizzi MC, Flegal KM, Dietz WH (2000) Establishing a standard definition for child overweight and obesity worldwide: international survey. Br Med J 320:1240-1243

9. Cole TJ, Flegal KM, Nicholls D, Jackson AA (2007) Body mass index cut offs to define thinness in children and adolescents: international survey. Br Med J 335:194

10. Cheuvront SN, Kenefick RW, Charkoudian N, Sawka MN (2013) Physiologic basis for understanding quantitative dehydration assessment. Am J Clin Nutr 97:455-462

11. Diethelm K, Huybrechts I, Moreno L, De Henauw S, Manios Y, Beghin L, Gonzalez-Gross M, Le Donne C, Cuenca-Garcia M, Castillo MJ, Widhalm K, Patterson E, Kersting M (2014) Nutrient intake of European adolescents: results of the HELENA (Healthy Lifestyle in Europe by Nutrition in Adolescence) Study. Public Health Nutr 17:486-497

12. Dinning A, Van SM, Wicken G (2005) Global socio-economic levels: development of a global non-occupational system. Int J Mark Res 47:597-614

13. Du S, Hu X, Zhang Q, Wang X, Liu A, Pan H, He S, Ma G (2013) Total drinking water intake and sources of children and adolescent in one district of Shenzhen. Wei Sheng Yan Jiu 42:433-436
14. Du SM, Hu XQ, Zhang Q, Wang XJ, Pan H, Gao JM, Song J, Gao CL, He ZF, Ma GS (2013) Daily intake of plain water and beverages of primary and middle school students in four cities of China. Zhonghua Yu Fang Yi Xue Za Zhi 47:202-205

15. Duffey KJ, Huybrechts I, Mouratidou T, Libuda L, Kersting M, De Vriendt T, Gottrand F, Widhalm K, Dallongeville J, Hallstrom L, Gonzalez-Gross M, De Henauw S, Moreno LA, Popkin BM (2011) Beverage consumption among European adolescents in the HELENA study. Eur J Clin Nutr 66:244-252

16. Edmonds CJ, Burford D (2009) Should children drink more water? The effects of drinking water on cognition in children. Appetite 52:776-779

17. Edmonds CJ, Jeffes B (2009) Does having a drink help you think? 6-7-Year-old children show improvements in cognitive performance from baseline to test after having a drink of water. Appetite 53:469-472

18. Feferbaum R, de Abreu LC, Leone C (2012) Fluid intake patterns: an epidemiological study among children and adolescents in Brazil. BMC Public Health 12:1005

19. Fenandez-Alvira JM, Iglesia I, Ferreira-Pego C, Babio N, Salas-Salvado J, Moreno LA (2014) Fluid intake in Spanish children and adolescents; a cross-sectional study. Nutr Hosp 29:1163-1170

20. Guelinckx I et al (2015) Intake of water and beverages of children and adolescents in 13 countries. Eur J Nutr. doi:10.1007/ s00394-015-0955-5

21. Kant AK, Graubard BI (2010) Contributors of water intake in US children and adolescents: associations with dietary and meal characteristics-National Health and Nutrition Examination Survey 2005-2006. Am J Clin Nutr 92:887-896

22. Nielsen SJ, Siega-Riz AM, Popkin BM (2002) Trends in food locations and sources among adolescents and young adults. Prev Med 35:107-113

23. Nowak M (1998) The weight-conscious adolescent: body image, food intake, and weight-related behavior. J Adolesc Health 23:389-398

24. Popkin BM, D’Anci KE, Rosenberg IH (2010) Water, hydration, and health. Nutr Rev 68:439-458

25. Senterre C, Dramaix M, Thiebaut I (2014) Fluid intake survey among schoolchildren in Belgium. BMC Public Health 14:651

26. Sichert-Hellert W, Kersting M, Manz F (2001) Fifteen year trends in water intake in German children and adolescents: results of the DONALD Study. Dortmund nutritional and anthropometric longitudinally designed study. Acta Paediatr 90:732-737

27. Silvaggio T, Mattison DR (eds) (1993) Comparative approach to toxicokinetics. Wilkins Wa, Baltimore

28. Stein AD, Shea S, Basch CE, Contento IR, Zybert P (1994) Assessing changes in nutrient intakes of preschool children: comparison of 24-hour dietary recall and food frequency methods. Epidemiology (Cambridge, Mass.) 5:109-115

29. Stookey JD, Brass B, Holliday A, Arieff A (2011) What is the cell hydration status of healthy children in the USA? Preliminary data on urine osmolality and water intake. Public Health Nutr 15:2148-2156

30. Tani Y, Asakura K, Sasaki S, Hirota N, Notsu A, Todoriki H, Miura A, Fukui M, Date C (2015) The influence of season and air temperature on water intake by food groups in a sample of free-living Japanese adults. Eur J Clin Nutr. doi:10.1038/ ejcn.2014.290

31. Thompson F, Subar A (2001) Dietary assessment methodology. In: A Coulston, CM Rock (eds) Nutrition in the prevention and treatment of disease. Monsen. Academic Press, San Diego, 3-22

32. Vergne S (2012) Methodological aspects of fluid intake records and surveys. Nutr Today 47:S7-S10 